\title{
Outcomes for offspring of men having ICSI for male factor infertility
}

\author{
Jane Halliday $^{1,2}$
}

Since the introduction of intracytoplasmic sperm injection (ICSI) using single sperm isolated from testicular tissue in men with obstructive and non-obstructive azoospermia, or using ejaculated sperm in those with poor semen quality, there have been concerns that this might have adverse effects on the offspring compared to conventional in vitro fertilisation (IVF) and natural conceptions. ICSI is done for reasons other than male factor infertility, and on the whole has not been shown to have any more negative effects than those seen with IVF. There have however, been very few studies of ICSI with a focus on, or large enough numbers to examine, the specific outcomes associated with male factor infertility. From the limited information available in relation to the source of the sperm and aetiology of infertility in the presence of ICSI, there appears to be no increased risk of congenital malformations. There is, however, a small increase in both de novo and inherited chromosome abnormalities. In terms of growth and neurodevelopment, there are very few studies, and so far, no adverse outcomes have been found in young children whose fathers have a sperm defect. The origin of the sperm used in ICSI does not have a major influence on the early life outcomes for the offspring, but transgenerational and epigenetic effects remain unknown. When the male factor infertility is known or thought to be due to a Y-chromosome deletion, this information should be given to the young male offspring at a time that will ensure his own reproductive health and plans are optimized.

Asian Journal of Andrology (2012) 14, 116-120; doi:10.1038/aja.2011.71; published online 12 December 2011

Keywords: congenital malformations; ICSI; male factor infertility; neurodevelopment; offspring outcomes; Y-chromosome deletions

\section{INTRODUCTION}

The use of intracytoplasmic sperm injection (ICSI), with both ejaculated and non-ejaculated sperm, circumvents natural selection barriers against sperm with physiological or genetic abnormalities. There is direct injection of the sperm into the vitellus of the oocyte during metaphase $\mathrm{II}^{1}$ which is technically invasive to the oocyte and may cause chemical or mechanical damage, such that errors occur in chromosome pairing because of disturbances of the meiotic spindle.

Although there are many articles written about outcomes associated with ICSI as a whole, as ICSI is being used increasingly in the absence of abnormal semen parameters or male factors, the role of male infertility per se in the analysis of outcomes has become less evident. There is often a masking of data pertaining to male-factor infertility as ICSI is being used to increase the odds of successful live birth in couples with not only male causes of infertility, but also cervical causes, or combined causes. ${ }^{2}$ The source of the sperm and type of infertility are seldom examined and the results are not stratified according to these potentially important exposures.

The major subgroups of male infertility, obstructive azoospermia (OA), non-obstructive azoospermia (NOA) and severe oligozoospermia (SO) seem likely to have different implications in terms of outcomes for the offspring. In the case of OA, there will be normal spermatogenesis but a mechanical problem preventing passage of the sperm, such as in men who have had damage to the epididymis because of infection or inflammation or have had vasectomies. OA can also be the result of congenital bilateral absence of the vas deferens in men with or without clinically evident cystic fibrosis. With NOA on the other hand, there is abnormal spermatogenesis as a result of testicular dysfunction. This is often due to genetic defects which affect production of sperm, such as Klinefelter's syndrome, Y-chromosome microdeletions and some translocations. ${ }^{3}$ For those with oligozoospermia, the actual sperm concentration is indicative of the potential presence of a genetic disorder, specifically a Y-chromosome microdeletion in those with $<1 \times 10^{6}$ sperm $\mathrm{ml}^{-1}$. ${ }^{4}$ Overall, there is variation in reports of the frequency of abnormal karyotypes in infertile men, most estimating between $8 \%$ and $10 \%{ }^{5-7}$ This variation persists when specifically looking for the Yq deletions, but it appears that $5 \%-10 \%$ of men with NOA or SO may have such a deletion. ${ }^{8}$ There are also many unrecognized genetic and unknown non-genetic causes of NOA and oligozoospermia.

Using the ICSI procedure, it is possible for males with OA, NOA and SO to father their own offspring using non-ejaculated sperm retrieved by microsurgical testicular sperm extraction (TESE) or epididymal sperm aspiration (MESA). A number of adverse outcomes have been postulated as a result of using sperm retrieved in these ways. First, TESE may result in extraction of immature sperm, which in turn might lead to an increased miscarriage rate and adverse effects on embryo development. ${ }^{9}$ This has in fact been refuted in a recent study which did not demonstrate an increased miscarriage rate in pregnancies resulting from TESE over and above that seen with MESA, both 
being $17 \%-18 \% .{ }^{10}$ Use of immature sperm may also result in imprinting errors associated with disruption of normal methylation erasure and re-establishment at critical stages of development. ${ }^{11-13}$ Another purported problem associated with the extraction of individual sperm is the potential use of aged epididymal sperm, which may lead to chromosomal errors. The third and most likely cause of adverse outcomes is the non-natural selection of a single sperm (ejaculated or non-ejaculated) which may lead to the passage of genetic defects that have caused the male infertility in the first place. ${ }^{4,14}$

It has been interesting to see over the years that most studies comparing ICSI with in vitro fertilisation (IVF) generally report no increased risks associated with ICSI over and above those associated with IVF in any particular outcomes for the offspring. ${ }^{15-19}$ There has been a recent study from Norway reporting that ICSI pregnancies have a lower risk of iatrogenic moderate preterm delivery, than IVF pregnancies. ${ }^{20}$ Although the preterm IVF pregnancies (8.6\% overall) had more congenital malformations than ICSI and may have contributed to the obstetric intervention ( $14 \%$ compared with $9.6 \%$ ), there is no convincing reason for this. In previous studies, an increased rate of preterm deliveries has not generally been reported as being significantly different.

In terms of obstetric outcomes, a retrospective cohort record linkage study from Australia in 2010 showed that singleton ICSI pregnancies have less adverse outcomes (post-partum haemorrhage, placenta praevia and antepartum haemorrhage) than IVF, although the difference is of borderline significance. ${ }^{21}$ This study highlighted the findings that are now emerging consistently, showing that adverse outcomes are most frequently associated with fresh embryo transfers in stimulated cycles as distinct from embryo transfers in natural menstrual cycles, as is the usual case with frozen-thawed embryo transfers. ${ }^{22}$ This may ultimately be a far more important predictor of outcomes than the IVF or ICSI technologies.

Although there are no marked differences between ICSI and IVF outcomes, the same differences between ICSI and spontaneous conceptions exist as between IVF and spontaneous conceptions. The overarching obstetric and perinatal concerns for all twin and higher order pregnancies remain for ICSI as well as for IVF pregnancies. ${ }^{23}$ Overall, compared with spontaneous conceptions, both ICSI and IVF are associated with lower birth weight, preterm delivery and an increased prevalence of congenital malformations. ${ }^{24,25}$ There have also been a variety of medical and psychosocial outcomes examined beyond the perinatal period demonstrating no specific differences between IVFand ICSI-conceived children in growth, ${ }^{26}$ neurological, ${ }^{27,28}$ cognitive ${ }^{29}$ and socio-emotional development ${ }^{30}$ and physical health. ${ }^{31}$

From here on, this review will present studies that have specifically examined aspects of male infertility, noting that there is limited published material available on offspring outcomes in the presence of male infertility. The outcomes of interest are congenital malformations overall and chromosomal defects specifically, the inheritance of male infertility as indicated by non-chromosomal markers and some sparse data on growth and neurodevelopment.

\section{CONGENITAL MALFORMATIONS}

\section{Overall defects}

Research into determining the risk of congenital malformations in babies conceived after IVF or ICSI has demonstrated clearly an overall increased risk, but is limited in terms of the ability to identify risks for specific birth defects, because of the inherent problem of small sample sizes. In addition, some studies do not report on prenatally diagnosed conditions for which there may be a termination as they have no record of events prior to 20 weeks of gestation; this creates a major underascertainment of real risks. Some believe there is overvigilance in clinical review of babies conceived by IVF or ICSI and therefore, overascertainment of less severe defects. Probably the biggest problems in comparing findings and coming up with meaningful risk figures are: the use of different definitions for congenital malformations, major versus minor; different coding systems; and different inclusion/exclusion criteria on the basis of such categorisation. We have recently published on another method of classification identifying congenital malformations (blastogenesis defects) that are most likely to be caused by events in the first 4 weeks of life. ${ }^{25}$ With all this in mind, there have been very few studies that have examined congenital malformations in relation to male infertility that provide convincing figures on the exact risks.

One of the first attempts to record congenital malformations associated with TESE and MESA came from the Brussels group, published in $1996 .{ }^{32}$ They found none among 29 children conceived using TESE, 1 out of 29 (3.4\%) MESA and 21 of 797 (2.6\%) standard ICSI. Such small numbers for the non-ejaculated sperm groups are almost meaningless in the context of congenital malformations and it has taken many years for numbers to build up at IVF centres and within registries so that comparisons can be made.

In 2001, a case-control study in the United Kingdom comparing rates of both major and minor abnormalities in 2-year-old offspring of oligozoospermic and non-oligozoospermic men was published. ${ }^{1}$ There was a large difference in overall rate of congenital malformations between the two groups, with the oligozoospermic group faring much worse ( 25 of $121,21 \%$ ) than the non-oligozoospermic group (7 of $87,8 \%)(P=0.02)$. Their data suggested that male urogenital problems may have been higher in the former group, but the authors stressed the need for larger registry-based studies.

Another study published in 2003, this time from Germany, focused on the source of the sperm (i.e., epididymal or testicular) and indication for ICSI. ${ }^{33}$ This study had slightly higher numbers, comparing outcomes for children conceived using 1785 ejaculated sperm with 147 testicular, but only 12 epididymal sperm. They reported no statistically significant difference in major malformations between different sperm sources, and in fact the only congenital malformations found in singletons were among the ejaculated sperm group (8.5\%). No individual malformations were analysed. They also examined their data by indication for ICSI, the vast majority being for severe idiopathic oligozoospermia, 68 for OA, 86 for NOA, 204 for failed IVF and 207 for other indications. They found no significant differences in risk of major malformations between the groups, with the OA rate being $8.4 \%$ and the NOA being $7.1 \%$.

A later (2005) study from Singapore stratified results on the basis of sperm count, motility and morphology for major malformations. ${ }^{34}$ They too, like the United Kingdom group, found slight evidence of an increase in adverse outcomes with severe oligozoospermia, but the numbers are far too small to place importance on.

In a European multicentre study reporting a follow-up of 3005 year-old children conceived using ICSI, including 266 whose fathers were infertile, a general statement was made that the rate of major malformations was no greater than that in the spontaneously conceived group, $14 / 252(5.6 \%)$ compared with $8 / 266(3.0 \%)$. In fact, there is almost a doubling in the odds ratio $(1.90 ; 95 \%$ confidence interval: $0.73-5.04$ ) and, with a larger sample size, this may have become significant. $^{35}$

A study from Norway (2007) analysed malformation data in children conceived using epididymal or testicular sperm and they 
observed an increased rate of hypospadias compared with the general population, with three boys out of 187 having hypospadias (1.6\%; 95\% confidence interval: $0.33 \%-4.7 \%){ }^{36}$ This study included both twins and singletons and the numbers were again very small. Male urogenital abnormalities have always been suspected of being increased because of the possible perpetuation via ICSI of fertility problems in the offspring of men with compromised spermatogenesis. Interestingly, however, important earlier studies of ICSI in general did not find such an increase $^{37}$ in male urogenital abnormalities and neither have more recent ones, ${ }^{38,25}$ Kallen et al..$^{38}$ specifically stated that 6 of $820(0.7 \%)$ infants born after non-ejaculated sperm ICSI had hypospadias, but it is not clear if this is higher than in the general population there.

A 2010 systematic review of five studies comparing the source of the sperm demonstrated no significant differences between the groupings in terms of risk for congenital malformations. ${ }^{39}$ The range of frequencies of congenital malformations in ejaculated sperm was from $1.9 \%{ }^{40}$ to $8.4 \%,{ }^{41}$ for epididymal sperm from $2.0 \%{ }^{40}$ to $4.3 \%{ }^{42}$ and for testicular sperm from $0 \%{ }^{42}$ to $9.2 \%{ }^{41}$ Within each study, there were no significant differences in risk of congenital malformations, although the range of frequencies reflects the inconsistencies mentioned earlier in regard to ascertainment of congenital malformations.

A study published this year has added considerably to knowledge of a number of outcomes for children conceived from either testicular or epididymal sperm, including congenital malformations. The Dutch multicentre study collected detailed information on a comparatively large number of children $(n=370)$ conceived with epididymal sperm. ${ }^{43}$ They compared outcomes for these 370 children with those conceived using ejaculated sperm in 1168 IVF and 1105 ICSI. No differences were observed in live and stillbirth rates, neonatal deaths or deaths up to 1 year of age. Neither were there any marked differences in rates of plurality, low birth weight, average gestation at birth or congenital malformations. The actual proportions of major malformations in 243 singletons were $3.3 \%$ in the epididymal group and $4.1 \%$ in the ejaculated ICSI group. An additional $8 \%$ and $12 \%$ of minor malformations were identified, respectively.

Another publication in 2011, from the Belgium cohort, compared outcomes for children conceived using testicular and epididymal sperm from 1994 to $2007 .^{44}$ The sample size was adequate to undertake meaningful statistical analyses, and built on an earlier publication. ${ }^{45}$ There were 512 offspring conceived using testicular sperm and 182 using epididymal sperm, and these were compared to each other and combined for a comparison with ejaculated sperm outcomes $(n=2477)$. There was a non-significant increase in prevalence of male genital abnormalities in the testicular/epididymal sperm groups combined $(0.7 \%)$ compared with the ejaculated sperm group $(0.3 \%)$, and this frequency is comparable to the general population. No differences existed for other congenital malformations in the two groups $(4.8 \%$ and $3.4 \%$, respectively), confirming the belief that the overall risk of congenital malformations does not depend on the origin of the sperm.

Congenital malformations that, because of their rarity, have not been able to be studied specifically in relation to male infertility are the imprinting syndromes. ${ }^{46}$ It is clear that, in the presence of IVF and ICSI, there is an increased risk of the ones caused primarily by epigenetic errors rather than chromosomal problems, such as the case with Beckwith-Weideman syndrome. ${ }^{47,48}$ The risk, albeit small, is important because it demonstrates the possibility of other, as yet unidentified, adverse outcomes due to epigenetic errors. Importantly in the context of this review, it does not appear to be an ICSI specific, and therefore, potentially male infertility-related, problem.

\section{Chromosomal defects}

The Belgium cohort has been studied carefully over the years on the basis of semen parameters and source of sperm, not only for overall congenital malformations, but also specifically for chromosomal abnormalities. An early follow-up of the ICSI-conceived children demonstrated an increase in de novo sex chromosome abnormalities in cases with SO in the father, but the actual proportions were not shown. A further study of this cohort published in 2002 (data from 1990 to 2001), on prenatally diagnosed chromosome abnormalities, suggested that the rate of de novo chromosomal abnormalities was higher in the testicular sperm group than in the ejaculated sperm group, $3.2 \%$ compared with $1.7 \%$, but the numbers were still small. ${ }^{49}$ They found no differences in the risks of inheriting a paternal chromosome abnormality and these were $1.6 \%$ and $1.4 \%$, respectively. There were no abnormalities found in the epididymal sperm group $(n=31)$.

The most recent study from the Belgium cohort (data from 1994 to 2007) showed that de novo chromosome abnormalities were present in $1.5 \%$ of the testicular sperm group, there were none in the epididymal sperm group and there were $1.7 \%$ in the ejaculated sperm group. ${ }^{44}$ Inherited chromosome abnormalities were present in $1.3 \%$ of the ejaculated sperm group and in $0.4 \%$ overall of the non-ejaculated sperm group. On this basis alone, the authors recommend, as had others before them, that when non-ejaculated sperm is to be used for ICSI, prenatal diagnosis is offered.

Comparable proportions come from a study in Turkey, published in $2004,{ }^{50}$ of abnormal karyotypes found prenatally in a testicular sperm group (128 karyotypes), compared to an ejaculatory sperm group (504 karyotypes). There was no statistical difference between the two groups, $1.5 \%$ and $1.9 \%$, respectively.

Y-chromosome deletions and the outcomes for boys inheriting a deletion are potentially problematic in terms of increasing the frequency of infertility in the general population. ${ }^{51}$ It is estimated that about 1 in every 1000 males has a Y-chromosome submicroscopic deletion rendering him infertile, but with the use of testicular sperm aspiration and MESA, their fertilisation or pregnancy rates are not decreased compared with non-Y-deleted men having ICSI. It is known that there is no increase in size of the deletion and the fertility problem will likely be the same as in the father. ${ }^{51}$

\section{Possible indicators of inherited male infertility}

Serum inhibin $B$. To further examine any possibility of male infertility in the father being passed on, 50 boys aged 8-14 years from the Belgium cohort have recently had their serum levels of inhibin B examined as a marker of potential oligozoospermia. ${ }^{52}$ Although the levels found were generally within the normal range, the length of follow-up and sample size are small. Therefore, this remains an important area of ongoing research, especially in light of another small study finding reduced serum testosterone levels in infant boys that correlated with their fathers reduced levels, but which has not apparently been confirmed or refuted by anyone else. ${ }^{53}$

Digit length and azoospermia. A study was designed to look for transmitted fertility problems in ICSI-conceived children who may show perturbations in finger development, thus reflecting the suggested genetic link between digit length and azoospermia. ${ }^{54}$ Digit measures were taken on 211 ICSI and 195 controls, and indeed shorter finger lengths than expected for their height were seen in both females and males conceived by ICSI. The importance of this in terms of fertility potential is not known. 


\section{GROWTH AND NEURODEVELOPMENT}

The Singapore cohort of ICSI-conceived children were analysed on the basis of sperm parameters in their fathers for differences in the Bayley scales measuring cognitive, language and motor development. ${ }^{34}$ They were approximately 2 years of age and, like another contemporaneous study using the Griffith scale of mental development, ${ }^{1}$ no problems were found in relation to sperm concentration, morphology or motility. In other words, the severity of the sperm defect in the father did not affect neurodevelopmental outcome in these young children.

In 2006, the European multicentre study examined the growth and cognitive development in 5-year-old children and revealed no differences in height and weight or IQ with epididymal and testicular sperm groups or with paternal sperm concentration. ${ }^{55}$

Most recently, the Dutch multicentre study cited earlier on 370 children conceived with epididymal sperm, ${ }^{43}$ had excellent response rates to a questionnaire sent to mothers and to an invitation for clinical review of children to 1 year and even some who reached 4 years of age in the study period. This allowed them to report on psychomotor development using the Bayley scales and again there were no differences found comparing outcomes to children conceived using ejaculated sperm in 1168 IVF and 1105 ICSI.

\section{CONCLUSIONS}

After careful review of the literature, it is evident that there is limited information available pertaining to offspring outcomes attributable specifically to male infertility and sperm parameters. Many of the studies have very small sample size for offspring conceived using epididymal sperm in particular. In summary, however, it appears that the origin of the sperm used in ICSI does not have a major influence on early-life outcomes for the offspring. The longer-term effects, such as transgenerational passage of male infertility through genetic or epigenetic processes, remain unresolved. It is recommended that genetic counselling should occur before treatment if there is a risk of transmitting a genetic defect, albeit very slight, and that embryo or fetal testing may also be 'appropriate in selected cases'. ${ }^{56}$ Such cases are those where there is use of non-ejaculated sperm for severe oligo- and azoospermia.

Boys who have been conceived using sperm from fathers with a Ychromosome deletion should be advised at a suitable age of their inherited infertility so that they can instigate their own reproductive planning process. The 'suitable' age may vary from child to child, and more research is needed to inform best practice in terms of age of disclosure of ICSI and particularly where there is a Y-chromosome deletion known or suspected.

Of course, the advice to parents having ICSI for any reason must include information about the risks of the well-described and -recognized adverse perinatal outcomes, particularly the increased risk of congenital malformations compared to spontaneously conceived children. This risk could be about $30 \%$ greater than the general population, giving a frequency of as high as $8 \%-10 \%$ depending on the categorisation of the malformations. It is probable that this information will seldom trouble the prospective parents enough to deter them from going ahead with ICSI, but at best they are doing so with some knowledge of the possible short-term risks. They must also understand that there is very little knowledge about the longer-term risks associated with assisted reproductive technology, especially in the presence of male infertility.

\section{ACKNOWLEDGEMENTS}

Associate Prof Halliday is funded by a National Health and Medical Research Council Senior Research Fellowship (No. 436904).

\section{COMPETING FINANCIAL INTERESTS}

The author declares no competing financial interests.

1 Sutcliffe AG, Taylor B, Saunders K, Thornton S, Lieberman BA et al. Outcome in the second year of life after in-vitro fertilisation by intracytoplasmic sperm injection: a UK case-control study. Lancet 2001; 357: 2080-4.

2 Nelson SM, Lawlor DA. Predicting live birth, preterm delivery, and low birth weight in infants born from in vitro fertilisation: a prospective study of 144,018 treatment cycles. PLoS Med 2011; 8: e1000386.

3 Georgiou I, Syrrou M, Pardalidis N, Karakitsios K, Mantzavinos T et al. Genetic and epigenetic risks of intracytoplasmic sperm injection method. Asian J Androl 2006; 8 643-73

4 Aittomaki K, Wennerholm UB, Bergh C, Selbing A, Hazekamp J et al. Safety issues in assisted reproduction technology: should ICSI patients have genetic testing before treatment? A practical proposition to help patient information. Hum Reprod 2004 19: 472-6.

5 Ferlin A, Arredi B, Foresta C. Genetic causes of male infertility. Reprod Toxicol 2006 22: $133-41$

6 Mohammed F, Al-Yatama F, Al-Bader M, Tayel SM, Gouda S et al. Primary male infertility in Kuwait: a cytogenetic and molecular study of 289 infertile Kuwaiti patients. Andrologia 2007; 39: 87-92.

7 Nagvenkar P, Desai K, Hinduja I, Zaveri K. Chromosomal studies in infertile men with oligozoospermia \& non-obstructive azoospermia. Indian J Med Res 2005; 122: 34 42.

8 Krausz C, Forti G, McElreavey K. The Y chromosome and male fertility and infertility. Int J Androl 2003; 26: 70-5.

9 Buffat C, Patrat C, Merlet F, Guibert J, Epelboin S et al. ICSI outcomes in obstructive azoospermia: influence of the origin of surgically retrieved spermatozoa and the cause of obstruction. Hum Reprod 2006; 21: 1018-24.

10 Kamal A, Fahmy I, Mansour R, Serour G, Aboulghar M et al. Does the outcome of ICSI in cases of obstructive azoospermia depend on the origin of the retrieved spermatozoa or the cause of obstruction? A comparative analysis. Fertil Steril 2010; 94: 2135-40.

11 Cox GF, Burger J, Lip V, Mau UA, Sperling K et al. Intracytoplasmic sperm injection may increase the risk of imprinting defects. Am J Hum Genet 2002; 71: 162-4.

12 Kobayashi H, Hiura H, John R, Sato A, Otsu E et al. DNA methylation errors at imprinted loci after assisted conception originate in the parental sperm. Eur J Hum Genet 2009; 17: 1582-91.

13 Palermo GD, Neri QV, Takeuchi T, Squires J, Moy F et al. Genetic and epigenetic characteristics of ICSI children. Reprod Biomed Online 2008; 17: 820-33.

14 Van Assche E, Bonduelle M, Tournaye H, Joris H, Verheyen G et al. Cytogenetics of infertile men. Hum Reprod 1996; 11(Suppl 4)1-24; discussion 25-6.

15 Kalra SK, Barnhart KT. In vitro fertilization and adverse childhood outcomes: what we know, where we are going, and how we will get there. A glimpse into what lies behind and beckons ahead. Fertil Steril 2011; 95: 1887-9.

16 Williams C, Sutcliffe A. Infant outcomes of assisted reproduction. Early Hum Dev 2009; 85: 673-7.

17 Steel AJ, Sutcliffe A. Long-term health implications for children conceived by IVF/ ICSI. Hum Fertil (Camb) 2009; 12: 21-7.

18 Alukal JP, Lipshultz LI. Safety of assisted reproduction, assessed by risk of abnormalities in children born after use of in vitro fertilization techniques. Nat Clin Pract 2008; 5: 140-50.

19 Sutcliffe AG, Ludwig M. Outcome of assisted reproduction. Lancet 2007; 370: 351 9 .

20 Morken NH. Preterm delivery in IVF versus ICSI singleton pregnancies: a national population-based cohort. Eur J Obstet Gynecol Reprod Biol 2011; 154: 62-6.

21 Healy DL, Breheny S, Halliday J, Jaques A, Rushford D et al. Prevalence and risk factors for obstetric haemorrhage in 6730 singleton births after assisted reproductive technology in Victoria Australia. Hum Reprod 2010; 25: 265-74.

22 Henningsen AK, Pinborg A, Lidegaard O, Vestergaard C, Forman JL et al. Perinata outcome of singleton siblings born after assisted reproductive technology and spontaneous conception: Danish national sibling-cohort study. Fertil Steril 2011; 95: 959-63.

23 van Steirteghem A, Bonduelle M, Devroey P, Liebaers I. Follow-up of children born after ICSI. Hum Reprod Update 2002; 8: 111-6.

24 McDonald SD, Han Z, Mulla S, Murphy KE, Beyene J et al. Preterm birth and low birth weight among in vitro fertilization singletons: a systematic review and meta-analyses. Eur J Obstet Gynecol Reprod Biol 2009; 146: 138-48.

25 Halliday JL, Ukoumunne OC, Baker HW, Breheny S, Jaques AM et al. Increased risk of blastogenesis birth defects, arising in the first 4 weeks of pregnancy, after assisted reproductive technologies. Hum Reprod 2010; 25: 59-65.

26 Basatemur E, Shevlin M, Sutcliffe A. Growth of children conceived by IVF and ICSI up to 12 years of age. Reprod Biomed Online 2010; 20: 144-9.

27 Knoester M, Vandenbroucke JP, Helmerhorst FM, van der Westerlaken LA, Walther FJ et al. Matched follow-up study of 5-8 year old ICSI-singletons: comparison of their neuromotor development to IVF and naturally conceived singletons. Hum Reprod 2007; 22: 1638-46.

28 Pinborg A, Loft A, Schmidt L, Greisen G, Rasmussen S et al. Neurological sequelae in twins born after assisted conception: controlled national cohort study. BMJ 2004; 329: 311 
Outcomes for offspring of male factor infertility

J Halliday

120

29 Ponjaert-Kristoffersen I, Bonduelle M, Barnes J, Nekkebroeck J, Loft A et al. International collaborative study of intracytoplasmic sperm injection-conceived, in vitro fertilization-conceived, and naturally conceived 5-year-old child outcomes: cognitive and motor assessments. Pediatrics 2005; 115: e283-9.

30 Barnes J, Sutcliffe AG, Kristoffersen I, Loft A, Wennerholm U et al. The influence of assisted reproduction on family functioning and children's socio-emotional development: results from a European study. Hum Reprod 2004; 19: 1480-7.

31 Bonduelle M, Wennerholm UB, Loft A, Tarlatzis BC, Peters C et al. A multi-centre cohort study of the physical health of 5-year-old children conceived after intracytoplasmic sperm injection, in vito fertilization and natural conception. Hum Reprod 2005; 20: 413-9.

32 Bonduelle M, Wilikens A, Burse A, van Assche E, Wisanto A et al. Prospective followup study of 877 children born after intracytoplasmic sperm injection (ICSI), with ejaculated epididymal and testicular spermatozoa and after replacement of cryopreserved embryos obtained after ICSI. Hum Reprod 1996; 11(Suppl 4): 13155; discussion 56-9.

33 Ludwig M, Katalinic A. Pregnancy course and health of children born after ICSI depending on parameters of male factor infertility. Hum Reprod 2003; 18: 351-7.

34 Agarwal P, Doh SK, Lim SB, Sriram B, Daniel ML et al. Two-year neurodevelopmental outcome in children conceived by intracytoplasmic sperm injection: prospective cohort study. Br J Obstet Gynaecol 2005; 112: 1376-83.

35 Bonduelle M, Burgh C, Niklasson A, Palermo GD, Wennerholm UB. Medical follow-up study of 5-year-old ICSI children. Reprod Boomed Online 2004; 9: 91-101.

36 Fodder J, Gabrielsen A, Humaidan P, Erb K, Ernst E et al. Malformation rate and sex ratio in 412 children conceived with epididymal or testicular sperm. Hum Reprod 2007; 22: 1080-5.

37 Hansen M, Kurinczuk JJ, Bower C, Webb S. The risk of major birth defects after intracytoplasmic sperm injection and in vito fertilization. N Eng J Med 2002; 346: 725-30.

38 Galen B, Finnstrom O, Lingam A, Nilsson E, Nygren KG et al. Congenital malformations in infants born after in vitro fertilization in Sweden. Birth Defects Res A Olin Mol Teratol 2010; 88: 137-43.

39 Woldringh GH, Besselink DE, Tillema AH, Hendriks JC, Kremer JA. Karyotyping, congenital anomalies and follow-up of children after intracytoplasmic sperm injection with non-ejaculated sperm: a systematic review. Hum Reprod Update 2010; 16: 12-9.

40 Palermo GD, Neri QV, Hariprashad JJ, Davis OK, Neck LL et al. ICSI and its outcome. Serin Reprod Med 2000; 18: 161-9.

41 Ludwig M, Katalinic A. Malformation rate in fetuses and children conceived after ICSI: results of a prospective cohort study. Reprod Boomed Online 2002; 5: 171-8.

42 Wennerholm UB, Burgh C, Hamberger L, Westlander G, Wikland M et al. Obstetric outcome of pregnancies following ICSI, classified according to sperm origin and quality. Hum Reprod 2000; 15: 1189-94.
43 Woldringh GH, Hovers M, Jansen AJ, Reuser JJ, de Groat SA et al. Follow-up of children born after ICSI with epididymal spermatozoa. Hum Reprod 2011; 26: 1759-67.

44 Belva F, de Schrijver F, Tournaye H, Liebaers I, Devroey P et al. Neonatal outcome of 724 children born after ICSI using non-ejaculated sperm. Hum Reprod 2011; 26: $1752-58$.

45 Vernaeve V, Bonduelle M, Tournaye H, Camus M, van Steirteghem A et al. Pregnancy outcome and neonatal data of children born after ICSI using testicular sperm in obstructive and non-obstructive azoospermia. Hum Reprod 2003; 18: 2093-7.

46 Amor DJ, Xu JX, Holiday JL, Francis I, Mealy DL et al. Pregnancies conceived using assisted reproductive technologies (ART) have low levels of pregnancy-associated plasma protein-A (PAPP-A) leading to a high rate of false-positive results in first trimester screening for Down syndrome. Hum Reprod 2009; 24: 1330-8.

47 Holiday J, Oke K, Breheny S, Alger EJ, Amor D. Beckwith-Wiedemann syndrome and IVF: a case-control study. Am J Hum Genet 2004; 75: 526-8.

48 Sutcliffe AG, Peters CJ, Bowdin S, Temple K, Reardon W et al. Assisted reproductive therapies and imprinting disorders - a preliminary British survey. Hum Reprod 2006; 21: 1009-11.

49 Bonduelle M, van Assche E, Joris H, Keymolen K, Devroey P et al. Prenatal testing in ICSI pregnancies: incidence of chromosomal anomalies in 1586 karyotypes and relation to sperm parameters. Hum Reprod 2002; 17: 2600-14.

50 Jozwiak EA, Slug U, Mesut A, Eden HF, Bahceci M. Prenatal karyotypes of fetuses conceived by intracytoplasmic sperm injection. Fertil Steril 2004; 82: 628-33.

51 Silver J. The $\mathrm{Y}$ chromosome in the era of intracytoplasmic sperm injection: a personal review. Fertil Steril 2011; 95: 2439-48.

52 Belva F, Bonduelle M, Painter RC, Schiettecatte J, Devroey P et al. Serum inhibin B concentrations in pubertal boys conceived by ICSI: first results. Hum Reprod 2010; 25: 2811-4.

53 Ma Kail C, Main KM, Andersen AN, Loft A, Skakkebaek NE et al. Reduced serum testosterone levels in infant boys conceived by intracytoplasmic sperm injection. J Chin Endocrinol Metal 2007; 92: 2598-603.

54 Sutcliffe AG, Manning JT, Katalanic A, Ludwig A, Meta M et al. Perturbations in finger length and digit ratio (2D:4D) in ICSI children. Reprod Boomed Online 2010; 20: $138-43$.

55 Wennerholm UB, Bonduelle M, Sutcliffe A, Burgh C, Niklasson A et al. Paternal sperm concentration and growth and cognitive development in children born with a gestational age more than 32 weeks after assisted reproductive therapy. Hum Reprod 2006; 21: 1514-20.

56 Practice Committee of the American Society for Reproductive Medicine. Does intracytoplasmic sperm injection (ICSI) carry inherent genetic risks? Fertil Steril 2004; 82( Suppl 1): S151-2.

Asian Journal of Andrology 